\title{
DIEZ POSTULADOS PARA UNA INVESTIGACIÓN CONTABLE UNIVERSITARIA DE CALIDAD. CONNOTACIONES ANTE EL PARADIGMA DEL VALOR EMPRESA
} TEN POSTULATES FOR HIGH QUALITY UNIVERSITY ACCOUNTING RESEARCH. IMPLICATIONS TO THE COMPANY VALUE PARADIGM

Gustavo Sader ${ }^{1}$

\section{Resumen}

Partiendo de las tres grandes visiones históricas de la universidad, se llega al análisis de la universidad argentina actual, donde la autonomía evaluada por el estado se centra en la búsqueda de la calidad. Ser docente e investigador universitario en el área contable implica conocer esa realidad y aceptar con sensatez las reglas, propiciando la calidad de sus producciones que hacen a su identidad. En el siguiente trabajo se proponen puntos de partida para la discusión sobre la calidad de los productos de la investigación contable universitaria, a través de la formulación de diez postulados, algunos de carácter epistemológico y otros de carácter metodológico. A modo de cierre, se ejemplifica con la problemática de la determinación del valor de una empresa y se observa la necesidad de complementar diferentes enfoques, ya que ninguno de los paradigmas vigentes pasa la línea de la valuación de una empresa entendida

1 Contador Público, Especialista en Sistemas Contables y Métodos Cuantitativos y Magíster en Sistemas de Información para la Toma de Decisiones. Docente e Investigador de las Universidades Nacionales de Río Cuarto (Profesor Titular Ordinario) y Villa María (Profesor Titular Ordinario). Categoría I en el Programa de Incentivos a los Docentes-Investigadores. Director de la revista científica académica Fundamentos de la FCE-UNRC. gusader@hotmail.com 
como la suma algebraica de sus componentes individuales, hacia la consideración de todos los elementos componentes para llegar al valor completo de la organización. Se entiende que es en este último punto donde las investigaciones de base teórica y empírica debieran complementarse.

Palabras clave: contabilidad financiera, epistemología, metodología, producciones científico-tecnológicas.

\section{Abstract}

Based on the three main historical perspectives on university research, we analyze today's university in Argentina, where autonomy as evaluated by the state focuses on the search for quality. Being a university professor and researcher in the accounting area implies sensibly accepting the rules whereby their identity is shaped by the quality of their work. The author proposes a list of starting points for discussing the quality of university accounting research work, through the formulation of ten postulates, some of which are epistemological and other, methodological. To conclude, the need to apply different approaches is exemplified with the problem of determining a company's value because current paradigms focus on the algebraic sum of the company's individual components without considering them as a whole. Therefore, both theoretical and empirical approaches should be adopted.

Keywords: Financial accounting, epistemology, methodology, scientific and technological work.

JEL: B49, M41. 


\section{Introducción}

Se parte de la historia de la universidad argentina hasta llegar al contexto actual, donde el Estado cumple un rol importante en la evaluación de la calidad educativa. Entendiendo como problema que la investigación universitaria, y la contable en particular, no se escapan de esta realidad, el objetivo se centra en proponer puntos de partida o postulados para mejorar la calidad de la investigación contable universitaria argentina.

Adicionalmente, a través de la determinación del valor empresa - un tema considerado paradigmático por el autor-, se muestra la importancia de mejorar la investigación por medio de pautas de calidad, ya que la relevancia de valor por sobre la medición y, por ende, aspectos cualitativos a revelar tienen igual importancia que su trascendencia numérica.

Figura 1. Estructura del artículo

Algo de Historia

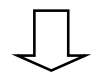

Somos investigadores-profesores universitarios y argentinos

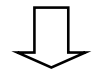

POSTULADOS

Epistemológicos

Metodológicos

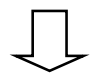

Connotaciones de la investigación contable ante el paradigma del valor empresa

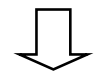

Conclusiones

Fuente: elaboración propia. 


\section{Algo de historia}

Para entender cuál es nuestra identidad como profesores e investigadores universitarios, es necesario conocer algo de historia, porque define nuestro contexto y da identidad a nuestra actual profesión. Particularmente: universitarios, contables y argentinos.

\section{Orígenes de la universidad}

En lo que respecta a la formación de la universidad moderna podríamos decir, siguiendo a Cubilla (2006: 1), que han influido tres grandes visiones históricas: la universidad de París, con énfasis en la formación integral humanista a través del uso de la lógica y el raciocinio; la universidad napoleónica, también de tradición medieval y difundida en Latinoamérica en una versión degradada, relaciona el aprendizaje con las necesidades inmediatas de la sociedad; y la universidad humboldtiana, originada en Berlín, cuya misión era la creación de conocimientos sin connotación utilitaria.

En lo que respecta a América Latina, la universidad comenzó siendo parte de un proceso de transferencia unilateral, producto, fundamentalmente, de un hecho administrativo que a la vez respondía a distintas orientaciones espirituales (Krotsch, 2001: 124). La universidad colonial latinoamericana es, entonces, una "universidad transferida" que solo logró enraizarse de manera débil en la realidad local de la que no había sido fruto. Luego de más de dos siglos las universidades comenzarán a desarrollar rasgos propios, poniéndose al servicio de las necesidades locales.

En este contexto, la universidad argentina parece no tener un origen determinado, en tanto, "no ha tenido un gesto fundante, un origen que pueda ser retenido en el tiempo como un acto de creación" (Krotsch, 2001: 126), y que determine su historia posterior, como es el caso de la universidad humboldtiana o napoleónica. Sin embargo, podría decirse que son los intereses de las profesiones liberales los que más fuertemente han influido en su conformación. En efecto, tal como señala Krotsch (2001: 187), la universidad argentina se desarrolla sobre la base de la incorporación aleatoria de distintos mode- 
los prevalecientes en el mundo que van siendo adaptados al estilo de demanda profesionalista.

\section{La universidad argentina. De la universidad colonial a la universidad del Estado}

La universidad colonial, con escasos recursos humanos y materiales y estudios fundamentalmente dirigidos a la formación de sacerdotes o agentes de la administración gubernamental, transita un proceso lento de inserción en el entramado de intereses de la región, dando paso, recién hacia a mediados del siglo XIX, a lo que se ha denominado "universidad de los abogados".

Esta institución, tal como expresa Krotsch (2001: 125), tendrá ahora como función no solo la de formar a jóvenes en las actividades que requerían las nuevas repúblicas - como las vinculadas a las armas, las profesiones liberales y la administración-, sino que debía hacerse cargo también del desarrollo del sistema educativo y, en algunos casos, alentar el desarrollo científico, tarea en la que no prosperará, pues las condiciones sociales no serán propicias para estas inquietudes especulativas.

En Argentina, las dos primeras universidades que surgen son la de Córdoba, creada en 1614 por los jesuitas, y la de Buenos Aires, que es creada más tardíamente, en 1821. La primera tuvo todas las características de las universidades de la Colonia y fue cuestionada, ya antes de la Independencia, por la creciente necesidad de formación especializada que requería la región del Río de la Plata. En cambio, la Universidad de Buenos Aires tuvo un desarrollo ligado más fuertemente a las necesidades de la sociedad en la que nace, que le exigió, antes que actividad científica real, el cumplimiento de ciertas funciones sociales. La Universidad de Buenos Aires respondió así al modelo de "universidad de los abogados", por su compromiso con la realidad social y económica de su región.

Alrededor de 1870 cobraron fuerza las ideas que vinculaban la misión esencialmente cultural y científica de la universidad con la autonomía universitaria y la desvinculación de la universidad de la función de otorgar títulos profesionales, que luego serán desnaturalizadas durante el proceso de nacionalización de las universidades (la Universidad de La Plata se nacionaliza en 
1905, la del Litoral en 1919 y la de Tucumán en 1921). Así, estas ideas solo se realizarán parcialmente en la educación superior argentina: la autonomía se combinará cada vez más con la construcción de una universidad de perfil profesionalista (Krotsch, 2001: 128-129).

Los estudios universitarios estaban destinados a la elite y la universidad era un espacio de prestigio y la antesala del poder, en tanto funcionaba armónicamente la relación entre el poder político y las universidades.

En todo el período que se extendió desde la Independencia hasta la Reforma de 1918, las universidades nacionales tendieron a concebirse como una prolongación del Estado.

\section{La Primera Reforma}

La Reforma de 1918, llamada Primera Reforma, constituye un proceso intelectual e institucional que se desplegó en la Argentina, y que extendió su influencia a casi toda América Latina. A partir del movimiento estudiantil nacido en Córdoba, que rechazaba el tradicionalismo católico prevaleciente, se gestó la lucha por la modernización de la universidad.

Esta reforma se caracterizó por su carácter endógeno, ya que las causas subyacentes de la Reforma estaban en la crisis de la estructura institucional y en la función social de la universidad.

Los principios de esta Reforma fueron el autogobierno, la representación de los estudiantes y graduados y la modernización de la enseñanza, acompañados de un compromiso con la cuestión social (Krotsch, 2001: 130). No obstante, en toda esta etapa y hasta comienzos de los cincuenta, el modelo napoleónico de universidad no había sido cuestionado en sus aspectos fundamentales y, en este sentido, la universidad continuó vinculada con las necesidades del Estado y con la formación de la elite ilustrada de la sociedad y de los profesionales liberales que demandaba la economía agroexportadora de la época. Asimismo, el número de universidades, en toda Latinoamérica, era escaso, la mayoría de ellas eran públicas y se localizaban en los centros metropolitanos. 


\section{La Segunda Reforma}

El período conocido como Segunda Reforma de la educación superior latinoamericana es aquel que se inicia al finalizar la Segunda Guerra Mundial y se extiende hasta la denominada "década perdida" (los ochenta).

Durante este período, la estrategia o forma general de actuación del Estado respecto de la educación superior fue el planeamiento (Cox, 1993: 115). Así, fue el Estado planificador en América Latina quien asumió funciones decisivas en el proceso de desarrollo y modernización de la región y en la transformación del modelo universitario tradicional (Krotsch, 2001: 133-134).

Se cuestiona, en esta etapa, el modelo napoleónico de universidad, la universidad de "elites", y se impulsa la modernización de la universidad a través de la adopción del modelo norteamericano, que involucraba la utilización de nuevos métodos pedagógicos, de nuevas formas de organización académicas (departamentos), el desarrollo de la investigación y la vinculación con la producción, entre otras innovaciones.

Esto fue acompañado por una multiplicación de las instituciones de educación superior, a través de la aparición de universidades privadas y de la creación de nuevas universidades nacionales. Asimismo, comenzó a producirse una fuerte diferenciación entre las instituciones tanto del sector público como del privado y la complejización tanto del sistema como de las instituciones mismas.

Estos procesos de diferenciación y complejización institucional fueron impulsados por el crecimiento de la demanda de educación superior que se había desarrollado como producto de la industrialización, la urbanización y la emergencia de nuevos sectores medios, lo cual fue determinante en el significativo aumento que tuvo la matrícula de educación superior en este período. Comienza a configurarse así una universidad de "masas" que deja de lado a la universidad de "elites".

La modernización general de la educación superior implicó también la aparición de carreras no tradicionales, la feminización de la matrícula, el crecimiento de la planta docente, la incorporación creciente de la investigación y la emergencia de la profesión de docente-investigador (Krotsch, 2001: 140). 


\section{La Tercera Reforma}

La década de los 90 se inició con una crisis fiscal, un producto per cápita que venía cayendo durante la década anterior, con la apertura de las economías hacia el exterior, en un marco de creciente globalización de los mercados. Estos cambios a nivel económico coinciden con el retorno de gobiernos democráticos. En este contexto, las relaciones entre el Estado, el mercado y la sociedad civil se transforman, y de sociedades fundadas en el Estado se intenta pasar a sociedades de mercado (Krotsch, 2001: 149-151).

En este contexto se da la Tercera Reforma de la educación superior en América Latina, en la que las nuevas políticas educativas toman como eje la descentralización de los servicios con el objetivo de mejorar la calidad de los mismos. La evaluación, que reemplaza a la planificación de la etapa anterior, se ubica en el centro de las nuevas políticas educativas y aparece como mecanismo complementario de los procesos de descentralización, a la vez que se propone como legitimadora de tales políticas.

Es por ello que se habla de que se produjo un "giro hacia la autonomía evaluada" que reemplaza la tradicional relación "benevolente" en materia de evaluación y asignaciones que había predominado en las décadas anteriores (Krotsch, 2001: 149-155).

Esta Reforma estuvo basada, fundamentalmente, en el desencanto con la situación vigente en la educación superior en América Latina después de treinta años de crecimiento del sistema, tanto en términos de instituciones como de matrícula. Así, hacia finales de los ochenta, se generalizan las críticas a la evolución de los sistemas de educación superior, principalmente, a nivel de los organismos internacionales, quienes tendrán un papel muy significativo en la construcción de la agenda de reformas de la educación superior.

Así, el Banco Mundial, cuyas propuestas se encuentran resumidas en Lessons of Experience (1994), señala que, en los países en desarrollo, será necesario alcanzar metas más altas de eficiencia, calidad y equidad. Y para ello plantea los siguientes objetivos:

- Promover la diferenciación de las instituciones, incluyendo el desarrollo de alternativas no universitarias y de instituciones privadas. 
- Proveer incentivos para que las instituciones públicas diversifiquen las fuentes de financiamiento, incluyendo aranceles y ligando el financiamiento estatal al desempeño.

- Redefinir el papel del Estado en la educación superior.

- Introducir políticas explícitamente orientadas al logro de la equidad.

Puede apreciarse, entonces, que durante este período el eje de las transformaciones ya no son las estructuras académicas o la orientación de las universidades, como en la etapa anterior, sino que el eje es el mercado y la reorientación en torno a esa lógica.

Además, esta reforma es conducida desde el exterior, por lo que se dice que es exógena y, además, global, por la amplitud del espectro de los cambios involucrados.

En la Argentina, fue a partir de 1987 y especialmente de 1993 que se introducen gran parte de las medidas de política que el neoliberalismo venía pregonando para el sistema universitario de la región. Los objetivos de esta política fueron los que el Banco Mundial había planteado en Lessons of Experience.

Durante el breve lapso comprendido entre 1993 y 1997 se formularon y comenzaron a implementar un conjunto de medidas que culminaron con la aprobación, en 1995, de la Ley de Educación Superior N.o 24521.

En términos generales, los cambios que se introdujeron se orientaron hacia el estímulo de la diferenciación institucional y a la competencia entre individuos e instituciones. En ese marco, el Estado, que se reservaba teóricamente un papel regulador y evaluador, va aumentando su carácter interventor, acrecentando los organismos y mecanismos de control y regulación de las instituciones. Se expande así la capacidad operativa del Ministerio de Educación y se crea un conjunto de organismos de amortiguación o espacios de mediación entre el Estado y las universidades, como el Consejo de Universidades, la Comisión Nacional de Evaluación y Acreditación Universitaria (CONEAU), los Consejos Regionales de Planificación de la Educación Superior, los cuales, junto con el Consejo Interuniversitario Nacional, el Consejo de Rectores de Universidades Privadas y la Secretaría de Políticas Universitarias (SPU), con- 
figuran el "gobierno" del sistema de educación superior, el cual va adquiriendo un creciente poder (Krostch, 2001: 165-166, 174, 182 y 185).

En síntesis, el espacio y el rol asignado a la investigación científica en las reformas llevadas adelante en el sistema educativo de nuestro país desde los años 90, considera dotar al rol docente de una nueva arista, incluyendo entre sus labores la de "investigar", asignación de una tarea que tradicionalmente no habían cumplido los docentes (Davini y Birgin, 1998).

Entonces, nuestro compromiso como docentes e investigadores es no estar ajenos a un contexto donde la evaluación de la calidad de nuestras producciones es importante. Esto no quiere decir que perdamos la sensatez y prevalezca la forma por sobre el contenido o la cantidad por sobre la calidad. Al menos, la comunidad científico-tecnológica actual requiere que nuestras producciones sean lógicas y coherentes e impliquen un aporte de calidad. Esta premisa también define nuestro ser profesor-investigador contable, universitario y argentino.

\section{Somos investigadores-profesores contables, universitarios y argentinos}

El sustantivo "profesor" y cada una de sus cualidades asociadas merecen una aclaración particular y, a su vez, el análisis de qué implican en su conjunto. En este sentido, la historia nos identifica como universitarios argentinos e invita entonces a la búsqueda de calidad. Por ende, es necesario entender el binomio docencia-investigación como actividades conexas que responden al perfil de calidad y hacen a la lógica del "ser universitario". De lo contrario, la universidad se limitaría solo a impartir enseñanza y no se diferenciaría de otro nivel educativo. La lógica que imponen estas afirmaciones está expuesta en Hernández Artiaga (2009):

Si se entiende a la investigación como una de las tareas centrales de la universidad, se puede entender que ésta constituya una de las actividades primordiales para el perfeccionamiento de la docencia, en tanto a través de ella (como proceso por el cual se desarrolla conocimiento mediante la indagación sistemática de la realidad en el 
marco del patrimonio científico y metodológico de la comunidad académica), es posible establecer la interrelación en el quehacer del proceso enseñanza-aprendizaje y la sociedad (p. 188).

Por otro lado, existe un maltrato generalizado del término "investigación en la docencia", pues se confunden ejercicios y notas académicas con indagación científica. Este problema puede llevar a posturas extremas entre el reconocimiento de la formación universitaria como un paso importante y necesario para la formación e incorporación de nuevos científicos y científicas, y el pesimismo por una educación que no forma investigadores e investigadoras en sentido estricto (Perkins, 2005).

Para ello es necesario que el investigador se forme, y la universidad deberá responder a esa demanda. En ese sentido, Mogollón (2007) expresa:

Existe la necesidad de diseñar propuestas para formar investigadores cuyas contribuciones deben proporcionar elementos innovadores que favorezcan la formación del investigador mediante la ampliación de experiencias asertivas, multidisciplinarias y éticas a través de las diversas actividades académicas que se ejecuten en el entorno universitario (p. 222).

Se plantea, entonces, un desafío. No solo debemos prepararnos para ser docentes universitarios, sino también para ser investigadores. Ahora, ¿qué implica ello en el ámbito de la contabilidad? Implica, en cierta manera, tener en cuenta la evolución epistemológica propia de nuestra disciplina, sus limitaciones, y la búsqueda constante de rigor metodológico en las producciones, en consonancia con los principios propios de la disciplina y de los principios que se manejan en la actualidad en el campo de las ciencias. Esta afirmación es independiente de considerarla como una disciplina científica o tecnológica, ya que en aras de generar nuevos conocimientos que redunden en beneficios a la sociedad, necesariamente se deberán utilizar parámetros científicos.

Es en este punto que el autor aporta postulados para su discusión, algunos de orden epistemológico y otros de orden metodológico, que responden, según su criterio, al ser docente-investigador universitario y contable en la actualidad. 


\section{Postulados}

En esta ponencia, la palabra "postulado" se sigue en la acepción de la Real Academia Española (RAE, 2017), como 'Idea o principio sustentado por una persona, un grupo, una organización, etc.', pretendiendo constituir un punto de partida o un ordenamiento de ideas para el análisis, en este caso, de la problemática de la investigación contable universitaria en la Argentina.

Siguiendo a Hernández Arteaga (2006), se pueden dividir en función al patrimonio que se pretende tutelar: "científicos", dando lugar en esta ponencia a postulados de corte epistemológico y a postulados de corte metodológico.

\section{Postulados epistemológicos}

POSTULADO 1. La contabilidad es ciencia en cuanto a la búsqueda de nuevos conocimientos que mejoren y amplíen su estructura lógica y su campo conceptual, y es tecnología en cuanto a su utilización en el campo de la realidad, atendiendo a la utilidad del usuario.

Según Beck (1998) como producto de la posmodernidad, la ciencia pasa de una fase de cientificación simple a una reflexiva.

En la fase simple se aplica la ciencia al mundo "dado" de la naturaleza, del hombre y de la sociedad; en la fase reflexiva, las ciencias ya están enfrentadas a sus propios productos, defectos, problemas inducidos; al pasar a la práctica se ve como producto y productora de la realidad y de los problemas que se ha encargado de analizar y dominar. De ahí que ya no resulta solo ser fuente de solución de problemas, sino que también a su vez es fuente que origina problemas. El mismo autor considera que en la práctica y en la opinión pública las ciencias se enfrentan, junto al balance de sus éxitos, al de sus fracasos y cada vez más al examen de sus promesas incumplidas.

La contabilidad no escapa de esa premisa. La comunidad científica formada por los investigadores contables se somete a una autocrítica de sus fundamentos y de su autocomprensión de manera pública. 
Así pues, la generalización de la ciencia reflexiva también abre, a los destinatarios y usuarios de la ciencia, nuevas posibilidades de influencia y desarrollo en los procesos de producción y aplicación de los resultados científicos. Este es un proceso de ambivalencia muy elevada: encierra la oportunidad de emancipación de la práctica social de la ciencia.

Lo que rige propiamente este proceso ya no es la cuestión de la verdad, sino la cuestión de la aceptabilidad social, la compatibilidad ética (Weingart, 1984).

Cuando la ciencia pasa a la práctica se enfrenta, junto al balance de sus éxitos, al balance de sus fracasos y al examen de sus promesas incumplidas, donde los destinatarios y usuarios de los resultados científicos no solo asumen una postura absolutamente crítica, sino que se convierten en activos coproductores de conocimiento. Así pues, la generalización de la ciencia reflexiva abre, para los destinatarios y usuarios de la ciencia, nuevas posibilidades de influencia y desarrollo en los procesos de producción y aplicación de los resultados científicos (Beck, 1998).

Esta es una realidad para la disciplina contable. Las ciencias económicas en general, y dentro de ellas la contabilidad, no se encuentran ajenas a este nuevo escenario, redefiniendo teorías, enfoques y perspectivas hacia saberes tecnológicos que orienten la práctica de la mejor manera posible.

Es en esta etapa reflexiva de la disciplina contable, asistimos a la confrontación con sus propios productos, defectos y problemas inducidos. Este pensamiento y esta reflexión incluye necesariamente el paradigma positivoutilitarista, donde los saberes científicos se conectan inevitablemente con los tecnológicos, ya que el logro de saberes tecnológicos es igual de importante que el de los saberes científicos.

En términos de productos de la investigación contable, se resalta la importancia de la diferenciación y confrontación de los saberes científicos -más relacionados con la disciplina madre: la economía, y con el método científico-y los saberes tecnológicos — que apuntan más a una demanda social ligada a la práctica-. Siguiendo las ideas de Beck (1998), es en ese punto donde la investigación contable se convierte tanto en producto como en productora de la realidad y de los problemas que se ha encargado de analizar y dominar. 
Muchos autores coinciden en la diferencia entre los saberes científicos y los tecnológicos. La definición de saberes científicos parte del concepto de ciencia. Siguiendo a Gamboa Gómez et al. (2012):

La ciencia es el conocimiento científico ordenado y mediato de los seres y sus propiedades, por medio de sus causas. El saber científico no aspira a conocer las cosas superficialmente, sino que pretende entender sus causas porque de esa manera se comprenden mejor sus efectos. Se distingue del conocimiento espontáneo por su orden metódico, su sistematicidad y su carácter mediato. Una aproximación crítica a la realidad apoyándose en el método científico fundamentalmente trata de percibir y explicar desde lo esencial hasta lo más prosaico el porqué de las cosas y su devenir, o al menos tiende a este fin (p.1).

Los saberes tecnológicos, por otro lado, se vinculan con la posibilidad de transformar la realidad, son más prácticos. Siguiendo a Solíverez (2013), la diferencia central entre las ciencias y las tecnologías es su función. Las principales funciones de las ciencias son la identificación, descripción, explicación, inferencia y predicción de fenómenos, aunque no siempre pueden cumplirse todas simultáneamente (en el campo social, las predicciones son casi imposibles). Las tecnologías, en cambio, son medios eficaces para la resolución de problemas prácticos, solo a veces de modo eficiente. Las ciencias permiten comprender el mundo; las tecnologías, transformarlo.

En la contabilidad se unen ambos conceptos. Es ciencia en cuanto a la búsqueda de mejorar y ampliar el marco conceptual y la estructura lógica de la disciplina; es tecnología en relación con sus productos y la utilización en la sociedad.

\section{POSTUlado 2. La dotación del camino epistemológico que encuadra el tema es fundamental.}

Este postulado es lógico, pero muchas veces no observado por los investigadores del área contable. En cierta manera, implica describir de manera resumida el camino epistemológico propio de cada autor y de la doctrina en gene- 
ral, que encuadran el tema de la investigación y su problemática. Es necesario contar desde dónde se parte y cómo surge la problemática que genera un ensayo, un artículo, una ponencia, una tesis o cualquier tipo de producción. En este sentido -y en la concepción tomada por el autor, considerando la contabilidad como ciencia social-, Vasilachis (2007) expresa:

Las ciencias sociales requieren, pues, encarar su particular reflexión epistemológica a partir de los desarrollos teóricos y de la práctica de la investigación empírica que les son propios. Esa reflexión, presente en la actividad del científico, aunque la lleve a cabo sin darle este nombre, está profundamente ligada a la elucidación de los paradigmas vigentes en la producción de cada disciplina (p. 134).

Vasilachis (1992) define estos paradigmas como los marcos teóricometodológicos utilizados por el investigador para interpretar los fenómenos sociales en el contexto de una determinada sociedad.

La propuesta es que postulados, conceptos, paradigmas, teorías y recortes de teorías sean incorporados completamente por los autores y con un correlato en el tiempo.

\section{POSTUlADO 3. En cualquier investigación, las producciones son siempre un comienzo, el conocimiento avanza lentamente y se genera un proceso de mejora continua.}

Este postulado es una invitación en doble sentido. Por un lado, invita a asumir que siempre estamos comenzando, que ninguna investigación se acaba en sí misma. Los esfuerzos de los doctorandos en terminar su tesis reivindican esta postura. Es un proceso largo, complejo, que pareciera terminar en la defensa de la tesis; sin embargo, al pasar el tiempo, se revela que la problemática no está acabada, que surgen nuevos caminos en la investigación.

Por otro lado, constituye una invitación a mantener las líneas de investigación en el tiempo. Los investigadores que hacen escuela raramente se adaptan a necesidades contextuales como muchas veces ocurre en la docencia universitaria argentina, donde los grupos de investigación cambian las líneas, solo por 
una cuestión de conveniencia. Esto es así ya que muchas veces los temas y problemáticas están "aparentemente" acabadas, y no surgen nuevas ideas. Otras veces, la incorporación de nuevos investigadores o necesidades del contexto, hacen cambiar la línea de investigación.

La importancia de permanecer en una línea implica conocer el propio proceso de investigación. La impaciencia no es una característica que debiera estar presente en los investigadores, y mucho menos en los docentes universitarios que investigamos en contabilidad. Los resultados se van generando en el tiempo, y nunca se termina el proceso. Cuando creemos acabado un estudio, surgen nuevos conceptos, hipótesis, relaciones que dan lugar a la mejora de posicionamientos, apertura de líneas correlacionadas, enfoques. Todo esto se debe dar en un contexto de multidisciplinariedad y del enfoque multiparadigmático $^{2}$ pretendido para nuestra disciplina

\section{Postulados metodológicos}

\section{POSTULADO 4. En un punto, la diferenciación entre investigación contable empírica e investigación contable teórica es innecesaria.}

Existe un proceso deductivo, pero necesariamente debe complementarse con lo inductivo, en términos de dar respuestas a la sociedad y generar un feedback que tienda a mejorar progresivamente la práctica contable.

\footnotetext{
2 Se adopta la concepción de "paradigma" de Belkaoui (2005), quien toma a la contabilidad como ciencia multiparadigmática, siguiendo al sociólogo Ritzer (1975) y a la propuesta de la AAA (1977). Este autor plantea para la contabilidad una serie de paradigmas, y amplía la concepción original de Kuhn (1962), que considera al paradigma como "un patrón, modelo de referencia o conjunto de reglas y procedimientos que son aplicados para la explicación de la realidad de un fenómeno o para la solución de un problema", y por ende, "un cambio de paradigma implica un cambio en los supuestos básicos". Según Kuhn (2010: 165), "una teoría científica solo se considerará inválida si hay un candidato alternativo para ocupar su lugar". Esta concepción, cerrada, es flexibilizada por Belkaoui (2005: 336) cuando expresa que "el paradigma sirve para diferenciar una comunidad científica (o sub-comunidad) de otra... define, e interrelaciona ejemplos, teorías, métodos e instrumentos que existen en su interior", concepto este más adaptado a las disciplinas sociales.
} 
Si bien a través de una investigación eminentemente teórica se pueden modificar cuestiones relacionadas con la estructura lógica y otros aspectos conceptuales, como el reconocimiento y medición de los elementos patrimoniales, en particular en esto último de alguna manera la empiria dará validez o no a los resultados de las investigaciones a través de la aceptación de los principios y criterios propuestos.

Lo que fortalece el proceso es el camino de ida y vuelta permanente entre lo deductivo y la empiria. Se puede hablar de una investigación teórica en cuanto implique la formulación de teorías o nuevos conocimientos, pero en algún punto tiene que existir una relación tal que permita mejorar la práctica.

Una división que se relaciona con esta problemática es la diferenciación entre investigación básica e investigación aplicada. Según Gómez Villegas (2003), por un lado, se encuentra la investigación básica, que se ocupa de las condiciones internas lógicas de la estructura conceptual de la teoría contable (teoría general) y de las teorías de sus aplicaciones, y de sus relaciones con otros campos del conocimiento, como la economía, la administración, el derecho, la sociología, la filosofía, entre otras. Versa sobre el estatuto epistemológico de la contabilidad. Su objetivo se centra en dar consistencia y rigor a las definiciones y estructuras contables buscando aumentar o validar el acervo conceptual de la disciplina y su relación transmulti- e interdisciplinaria. Por otro lado, existe un tipo de investigación más concerniente a las necesidades instrumentales y funcionales del entorno en el que opera la contabilidad, es decir, a las necesidades de información, control, medición y representación de la riqueza en organizaciones específicas como la empresa, el sector gubernamental y la nación, entre otros, que se denomina "investigación aplicada". Esto da lugar a pensar que existe una relación entre la investigación básica y la teórica y entre la investigación aplicada y la empírica.

Desde un punto de vista teleológico, el autor considera innecesaria la división que hace la doctrina. Hablar de investigación contable es más sensato. Cuando se habla de investigación empírica, necesariamente se incluye la teoría, ya que no debería existir una investigación empírica sin fundamentos en un marco teórico que la sustente. Ahora, cuando se habla de investigación 
teórica, no se hace referencia a la empírica, salvo a algunos estudios de corte empírico, aunque la finalidad de la contabilidad, tenga un alto sentido social.

Es importante que esta diferenciación no divida a los investigadores contables. La diferencia no es tan tajante como parece. No es posible considerar la una sin la otra, ya que, en algún punto, al tratarse de una disciplina con alta connotación tecnológica, ambas se relacionan.

\section{Postulado 5. No deben existir resultados de investigación que no se pre- senten con cierto rigor metodológico, ya que los problemas de forma mu- chas veces impiden apreciar el fondo.}

Una investigación de alta calidad se caracteriza por varios atributos distintos (Salkind, 1999), a saber: se basa en el trabajo de otros, se puede repetir y generalizar a otras situaciones, se basa en algún razonamiento lógico y está vinculada a una teoría, además, se puede hacer (construir), a partir de ella se pueden generar nuevas preguntas, es de naturaleza cíclica, es incremental y debe ser una actividad apolítica que debe emprenderse con el fin de mejorar la realidad.

Ese razonamiento lógico-deductivo ayuda al objetivo de calidad científica pretendido, y en las producciones está dado por la búsqueda de cierto rigor científico o rigor metodológico, el que supone:

- El respeto de una estructura secuencial lógica que involucre los pasos del proceso de investigación científica (idea y tema, problema, marco teórico, tipo de investigación, hipótesis-preguntas de investigaciónobjetivos, definición de variables, diseño, muestra y análisis de datos, resultados, discusión y conclusiones). Sin embargo, no todos estos pasos son necesarios en la investigación contable y van a depender del diseño de investigación que se pretenda encarar, con base en la mejor manera de resolver el objeto problema planteado por la investigación.

- El cumplimiento de al menos cuatro "relaciones críticas" en el trabajo de investigación que le otorgan "calidad científica", a saber: 1) entre los objetivos generales y el título, 2) entre las conclusiones y los objetivos (las conclusiones dan respuesta a los objetivos), 3) entre las conclusiones y los resultados (las conclusiones deben estar respaldadas en resultados, 
y 4) entre las conclusiones y el objeto problema (las conclusiones resuelven el objeto problema).

\section{Postulado 6. En una investigación de base empírica el análisis de datos es sólo una parte del proceso. No descuidar las otras.}

El camino en una investigación empírica es lógico pero arduo. Se parte de la concepción de la idea y la motivación, siguiendo por el planteamiento del problema, las preguntas de investigación, la formulación de hipótesis, los objetivos generales y específicos, la definición del tipo de investigación, el diseño de la misma, el tratamiento de los datos y herramientas estadísticas, hasta llegar a la interpretación de resultados y las conclusiones.

Se advierte que, en este proceso, se le da mucha importancia al tratamiento de los datos - y a las herramientas estadísticas- para decodificarlos y luego interpretarlos. A tal punto es así que muchos de los cursos de metodología que se dan en los posgrados se limitan solo a esta parte del proceso.

Los cuestionamientos a esta manera de encarar la faz metodológica de las producciones son lógicos, ya que si no se parte de un problema general, se delimita el objeto de estudio, se trazan los objetivos, se da respuesta a ellos partiendo de las preguntas de investigación o de las hipótesis, con una total coherencia en todo el proceso, el tratamiento y el análisis de datos, entonces no se cumple con la coherencia lógica que requiere el proceso.

El autor, ha analizado trabajos en donde la herramienta estadística se muestra más poderosa que los objetivos de investigación, y los resultados no son congruentes con el planteamiento y resolución del problema de investigación.

\section{PostulAdo 7. La redacción de las producciones debe ser clara y secuencial.}

Según Azzolini y Nistal (2017), desde ese rol docente, el poder aplicar los conocimientos que se adquieren en el desarrollo de una pregunta científica, demanda la necesidad de conocer y dominar la forma de comunicar conocimientos a través de un texto científico. 
Para ello, desde el comienzo, se debe saber de manera sintética y efectiva a qué se llega. Comenzando ya en el resumen, se deben marcar el porqué, el cómo y el dónde se pretende llegar. Para ello, es necesario no solo una clara redacción, sino una secuencia que lleve a las conclusiones de una manera amena para el lector, adelantando en todo el texto cómo se arriba a estos resultados.

\section{POSTULADO 8. Es importante empezar cualquier producción aportando la "motivación" del/os autor/es.}

La "motivación" según el diccionario de la RAE (2017), es 'Acción y efecto de motivar'. La causa es el motivo: conjunto de factores internos o externos que determinan en parte las acciones de una persona, en este caso, el autor. La motivación, por ende, depende de factores o intereses personales, pero también del contexto de investigación. Implica, en cierta manera, responder ¿por qué investigamos?

La importancia de su inclusión en los textos de investigación contable es para dar a entender características propias del investigador (motivación), particularizar la investigación en la persona o grupo de investigadores, e, incluso para dar cuenta de factores relacionados con la productividad (González Blasco, 1979).

\section{Postulado 9. Cualquier investigación contable debiera partir de una idea, de la definición del problema general y su delimitación en función de los objetivos.}

Según Hernandez Sampieri et al. (2003), una vez concebida la idea de investigación, se está en condiciones de plantear el problema de investigación. Plantear el problema no es sino afinar y estructurar más formalmente la idea de investigación. El paso de la idea al planteamiento del problema en ocasiones puede ser inmediato, casi automático, o bien llevar una considerable cantidad de tiempo; ello depende de cuán familiarizado esté el investigador con el tema a tratar, la complejidad misma de la idea, la existencia de estudios antecedentes, el empeño del investigador, el enfoque elegido (cuantitativo, cualitativo o mixto) y sus habilidades personales. 
Seccionar un tema o una idea no coloca al investigador inmediatamente en la posición de considerar qué información habrá de recolectar, con cuáles métodos trabajará y cómo analizará los datos que obtenga. Antes necesita formular el problema específico en términos concretos y explícitos, de manera que sea susceptible de investigarse con procedimientos científicos (Selltiz et al., 1980). En el caso del enfoque cualitativo en la investigación, o cuando este enfoque prevalece sobre el cuantitativo, el planteamiento del problema llega a tener lugar en diferentes momentos de la investigación: puede seguir a la idea, surgir durante la investigación o presentarse al recolectar la información.

Carrasco (2009) sostiene que para plantear el problema de investigación se debe tener en cuenta lo siguiente:

- Tener conocimiento amplio y objetivo del tema y los problemas de investigación, es decir, conocer cómo se manifiesta el problema de investigación y cómo afecta al contexto social —dicho de otro modo, cómo ha surgido y ha evolucionado-.

- La formulación que es parte del planteamiento del problema debe hacerse en preguntas, vale decir, aun cuando se puede formular el problema de manera afirmativa, es conveniente hacerlo como interrogación, puesto que expresa mayor viabilidad metodológica.

- Es recomendable incluir la justificación del estudio en el planteamiento del problema, puesto que la justificación en una investigación proporciona los fundamentos del porqué de la investigación. Esto constituye un elemento de consistencia y de motivación para el investigador.

- El problema debe estar debidamente delimitado. Esto permite prescindir de ambigüedades respecto del tiempo, espacio y marco teórico.

- Los elementos fácticos y metodológicos que conforman el planteamiento del problema de investigación, deben guardar relación y coherencia entre sí. 
POSTULADO 10. En la investigación contable, no siempre es necesario formular hipótesis; con una enumeración de preguntas de investigación relacionadas con el objeto problema, se resuelve su ausencia.

Además de definir los objetivos concretos de la investigación, es conveniente plantear a través de una o varias preguntas —según sea el caso- el problema que se estudiará. Plantear el problema de investigación en forma de preguntas tiene la ventaja de presentarlo de manera directa, minimizando la distorsión (Christensen, 1980).

No es necesario plantear hipótesis en todas las investigaciones. La experiencia muestra que se hallan producciones teóricas con hipótesis donde evidentemente no existe un proceso que conlleve a su aceptación o su rechazo.

Siguiendo a Hernandez Sampieri et al. (2010), en las investigaciones cuantitativas, cuyo método es el deductivo sí es necesario formular hipótesis, siempre y cuando se defina desde el inicio que su alcance será correlacional o explicativo, o, en caso de un estudio descriptivo, que intente pronosticar una cifra o un hecho. En cambio, en los estudios cualitativos, generalmente no se formulan hipótesis antes de recolectar datos. Su naturaleza es más bien inductiva, lo cual es cierto, particularmente, si su alcance es exploratorio o descriptivo. Desde luego, cuando su alcance es correlacional o explicativo es posible formular hipótesis durante la obtención de la información, después de recabar datos, al analizarlos o al establecer las conclusiones.

\section{Connotaciones de la investigación contable ante el paradigma del valor empresa}

Ante esta problemática, la investigación en contabilidad se encuentra en un período de preciencia, ya que el interés en determinar el valor de una empresa ha generado nuevas necesidades de información que la contabilidad deberá resolver. En este sentido, ninguno de los paradigmas vigentes pasa la línea de la valuación de una empresa considerada como la suma algebraica de sus componentes individuales, hacia la consideración de todos los elementos 
componentes, para llegar al valor completo de la empresa, o sea, incluyendo el denominado capital intelectual.

El actual período que atraviesa la investigación contable también puede considerarse -por los motivos ya expuestos- como de ciencia normal alarmante, ya que se visualiza un notable avance hacia la normalización internacional. Sin embargo, en el nivel teórico surgieron solo algunos conceptos y herramientas no muy desarrollados, complementados por la coexistencia de los mismos paradigmas a través del tiempo, generando una sinergia entre ellos que no logra romper la inercia para avanzar hacia nuevos paradigmas.

En este sentido, las investigaciones sobre empresas que cotizan en bolsa son particularmente fructíferas, ya que se cuenta con la visión y reacción del usuario colectivo ante la viabilización de una teoría a través de la norma y que se refleja inmediatamente en la diferencia entre el valor de cotización de las acciones respecto de su valor contable (market to book). Entonces, la distancia que existe entre este valor y el valor contable del patrimonio neto es posible de transitar a través de la investigación contable, tratando de identificar las causas que la generan. En esta línea, el autor y un equipo de investigación están abocados desde hace una década.

Por otro lado, la disciplina contable solo estuvo en su faz simple de cientificación cuando a la luz de un enfoque legalista y técnico, con base en la partida doble, reflejó la situación y evolución del patrimonio y sus resultados en base al costo histórico; el núcleo del paradigma se centró específicamente en mostrar la instrumentación jurídica de las operaciones por el monto de la inversión original, más allá de algunos intentos de incluir criterios relacionados con la tributación y sus leyes. Luego pasó a una fase reflexiva derivada del desarrollo de los negocios, de las teorías sobre valor y precios y la irrupción de procesos inflacionarios. La disciplina contable empieza a cuestionar sus propias bases y surgen así métodos de corrección del costo (donde la inversión original queda expresada en moneda homogénea del momento de la medición, aunque prevalezca el mismo criterio); y la incorporación de precios corrientes basados en un mercado de entrada o de salida según los atributos de los elementos patrimoniales a cuantificar; o de algunos valores como las revaluaciones técnicas o el valor patrimonial proporcional, que más que un valor es una técnica de valora- 
ción de una clase de inversiones. No obstante, no se supera la línea de la medición de los elementos patrimoniales, ya que el valor empresa hasta ese momento solo era un concepto muy difuso, y si bien se ha avanzado al respecto, las propuestas doctrinarias no parecen proponer una salida hacia nuevos paradigmas, y por ende, hacia un avance científico o tecnológico.

En tal sentido, la discusión sobre si los productos de la disciplina contable son saberes científicos o tecnológicos asume una respuesta dual. Se consideran científicos en cuanto respeten bases teóricas existentes y aceptadas por las comunidades científicas y cierto rigor metodológico; y tecnológicos en cuanto den respuesta a problemas prácticos.

Pero ¿es el paradigma del valor empresa el punto de inflexión entre la ciencia normal y el surgimiento de nuevos paradigmas? La respuesta a esa pregunta es el punto de partida desde donde los investigadores debemos comenzar a avanzar. Y para ello, seguir un proceso coherente, con rigor metodológico, de base deductiva-inductiva y con productos de calidad nos conducirá a la tan pretendida "calidad", y nos ayudará a mejorar nuestra identidad como investigadores universitarios. Si en la universidad, el conocimiento no se genera de esta manera, ¿en qué otro ámbito será posible?

\section{Conclusiones}

Diferentes divisiones ponen la contabilidad en el camino de la ciencia, ya que la misma puede asumir desde su campo epistemológico, como desde su producto tecnológico, diversas orientaciones. Así, las dualidades planteadas por diversas clasificaciones —como ciencia básica y aplicada, productora de saberes científicos y tecnológicos, en etapa de cientificación simple o reflexiva, investigación teórica y empírica- nos lleva a reflexionar sobre la generación de sus bases conceptuales y la aplicación en el campo de la realidad.

Desde el punto de vista investigativo, todas las dualidades están relacionadas, y contribuyen al avance de esta disciplina. Según el autor, en la problemática de la determinación del valor de una empresa es donde se evidencia la necesidad de complementar todos los enfoques, ya que ninguno de los paradigmas vigentes pasa la línea de la valuación de una empresa tenida en cuenta 
como la suma algebraica de sus componentes individuales, hacia la consideración de todos los elementos componentes para llegar al valor completo de la empresa, o sea, incluyendo el denominado capital intelectual

Se propone la discusión de los postulados enunciados, considerando que constituyen un buen punto de partida para la investigación contable en general, en términos de mejorar la calidad de las producciones y, en particular, para las investigaciones sobre la medición del capital intelectual.

\section{Referencias bibliográficas}

Azzolini, S. \& Nistal, M. (2017). Pautas para la elaboración de un texto científico. Recuperado el 29/09/17 de http://www.colegiomilitar.mil.ar/rediu/pdf/ ReDiU_0924_art3-texto_cientifico(Azzollini_Nistal).pdf

AAA (American Accounting Association) (1977). Statement of Accounting Theory and Theory Aceptance (SOATATA). Committee to prepare a Statement of basic Accountant Theory.

Banco Mundial (1994). Higher Education: Lessons of Experience. Washington, D.C. World Bank.

Beck, U. (1998) ¿Ciencia más allá de la verdad y de la ilustración? Reflexividad y crítica del desarrollo científico y tecnológico. En La sociedad del riesgo. $\mathrm{Ha}$ cia una nueva modernidad (203-235). Buenos Aires: Paidós.

Belkaoui, A. (2005). Accounting Theory. (5. a ed.). Chicago, Illinois: United States of America.

Brunner, J. (2005). Tendencias Recientes de la Educación Superior a Nivel Internacional: Marcopara la Discusión sobre Procesos de Aseguramiento de la Calidad. Escuela de Gobierno, Universidad Adolfo Ibáñez, Santiago de Chile.

Carrasco Díaz, S. (2009). Metodología de Investigación Científica. Lima: Editorial San Marcos, p. 93.

Cox, C. (1993). Políticas de Educación Superior: Categorías para su Análisis. En H. Corard (ed.). Politicas Comparadas de Educación Superior en América Latina. Santiago de Chile: FLACSO.

Christensen, L. B. (1980). Experimental methodology (2. a edición). Boston, Mass.: Allyn and Bacon, Inc. 
Cubilla, A. (2006). Reforma Universitaria: Visiones Múltiples y Dos Acciones para el Cambio. Recuperado en marzo de 2010 del sitio Web del Corredor de las Ideas: www.corredordelasideas.org/docs/reflexion_universitaria.doc

Davini, M. C. \& Birgin, A. (1998). Políticas de Formación Docente en el escenario de los '90. Continuidades y rupturas. En AA. VV. Politicas y sistemas de formación. Buenos Aires: Novedades Educativas/Facultad de Filosofía y Letras de la UBA.

Hernández Sampieri, R., Fernández Collado, C. \& Baptista Lucio, P. (2010). Metodología de la Investigación. México: Mc. Graw Hill.

Krotsch, P. (2001). Educación Superior y Reformas Comparadas. Buenos Aires: Universidad de Quilmes.

Kuhn, T. (1962, 2010). La estructura de las revoluciones científicas. México: Fondo de Cultura Económica.

Mogollón, A. (2007). Formación del investigador universitario. Revista Ciencias de la Educación, año 6 1, 1(29), Valencia, 217-230

Gamboa Gómez, A., Hormiga, M., Zambrano, L. \& Collo Chávez, L. C. (2012). Epistemología. Trabajo colaborativo No. 1. Aporte individual. Universidad Nacional Abierta y a Distancia. Escuela de Ciencias Administrativas, Económicas y de Negocios. Popayán. Colombia. En http://epistemologiacienci.blogspot.com.ar/

Gómez Villegas, M. (2003). Algunos comentarios sobre la potencialidad de la investigación en contabilidad. INNOVAR. Revista de Ciencias Administrativas y Sociales, enero-junio, 139-144.

González Blasco, P. (1979). Motivación y productividad en la investigación científica española Revista Española de Investigaciones Sociológicas, 69-75.

Hernández Artiaga, I. (2009). El docente investigador como creador de conocimiento. Revista Ciencias Biológicas Tumbaga, 4, 185-198.

Perkins, D. (2005). La enseñanza para la comprensión: cómo ir de lo salvaje a lo domesticado. Revista magisterio, 14, 10-15.

RAE (2017). Diccionario de la Real Academia Española. Versión online, Recuperado el 24/09/17 de http:/ / dle.rae.es/

Ritzer, G. (1975). Sociology: A Multiple Paradigm Science. Boston: Allyn and Bacon. 
Rojas Betancur, H. M. (2009). Formar investigadores e investigadoras en la universidad: optimismo e indiferencia juvenil en temas científicos. Revista Latinoamericana de Ciencias Sociales, Niñez y Juventud [en línea], 7 (juliodiciembre). Recuperado el 11/09/2017 de http://www.redalyc.org/ articulo.oa?id=77314999018

Salklind, N. J. (1999). Metodología de la Investigaciónk (3. a ed.). México: Prentice Hall.

Selltiz, C., Mahoda, M., Deutsch, M. \& Cook, S. W. (1980). Métodos de investigación en las relaciones sociales. Madrid: Ediciones Rialp.

Solivérez, C. E. (2013). ¿De qué hablan los argentinos cuando hablan de tecnología? Revista Tekné, 3, Facultad de Arte y Diseño, Universidad Nacional de Misiones, 17-19.

Vasilachis de Gialdino, I. (1992). Métodos cualitativos. Los problemas teóricoepistemológicos. Buenos Aires: Centro Editor de América Latina.

Vasilachis de Gialdino, I. (2007). El aporte de la epistemología del sujeto conocido al estudio cualitativo de las situaciones de pobreza, de la identidad y de las representaciones sociales. Forum Qualitative Sozialforschung/ Forum: Qualitative Social Research, 8(3).

Weingart, P. (1984). The structure of technological change: reflections on a sociological analysis of technology. En E. Laudan (ed.). The nature of technological knowledge. Are models of scientific change relevant? Dordrecht: D. Reidel, 115-142.

(C) 2017 por los autores; licencia otorgada a la revista Escritos Contables y de Administración. Este artículo es de acceso abierto y distribuido bajo los términos y condiciones de una licencia Atribución-No Comercial 4.0 Internacional (CC BY-NC 4.0) de Creative Commons. Para ver una copia de esta licencia, visite https://creativecommons.org/licenses/by-nc/4.0/ 